[0212-7199 (2005) 22: 4; pp 191-193] ANALES DE MEDICINA INTERNA Copyright (C) 2005 ARAN EDICIONES, S.L

AN. MED INTERNA (Madrid) Vol. 22, N. ${ }^{\circ} 4$, pp. 191-193, 2005

\title{
Osteomielitis esternal hematógena y neumonía de la comunidad secundarias a una sepsis por Staphylococcus aureus sensible a meticilina
}

\author{
Y. TORDECILLA ECHENIQUE, M. P. SALAMANCA BAUTISTA, J. L. ARIAS \\ JIMÉNEZ, E. GUISADO ESPARTERO, M. ORTEGA CALVO ${ }^{1}$, R. PÉREZ CANO
}

Servicio de Medicina Interna. Hospital Universitario Virgen Macarena. Sevilla. ${ }^{\text {} C e n t r o ~}$ de Salud Esperanza Macarena. Sevilla

HAEMATOGEOUS STERNAL OSTEOMYELITIS AND COMMUNITY PNEUMONIA IN A METICILIN-SUSCEPTIBLE STAPHILOCOCCUS AUREUS SEPSIS

\section{RESUMEN}

Presentamos un caso de osteomielitis esternal hematógena en un varón joven con antecedentes de asma moderado y normalidad de las funciones inmunológicas. El cuadro se desencadenó después de un esfuerzo físico importante y probablemente por la reactivación de una lesión foruncular en la pared del abdomen. Se aisló Staphylococcus aureus sensible a meticilina tanto en los hemocultivos como en el líquido extraído de la lesión esternal. Se pudo objetivar una elevación de la fracción Ig G 4 en la fase de reimisión clínica. El cuadro séptico se acompañaba de lesiones pulmonares sugerentes de neumonía neumocócica. El tratamiento a base de cloxacilina y gentamicina por vía endovenosa seguido por Rifampicina y Levofloxacino por vía oral dio como resultado una "restitutio ad integrum".

PALABRAS CLAVE: Sepsis. Osteomielitis esternal. Neumonía de la comunidad. Estafilococo dorado. Asma bronquial extrínseco.

\begin{abstract}
We report here a case of primary haematogenous osteomyelitis diagnosed in a young mild asthmatic male with immunocompetence. A hard job worked as trigger of the septic picture from a forunculosis lesion located on the abdominal wall. Meticilin-susceptible Staphylococcus aureus was isolated from blood cultures and from sternal aspiration liquid.

Two months after clinical onset Ig G 4 elevation was achieved at the immunodeficiency screening. Stafilococycal lung CT images accompanied to the septic course. Intravenous cloxacilin and gentamycin treatment followed by oral rifampicin and levofloxacin achieved a total recovery.
\end{abstract}

KEY WORDS: Sepsis. Sternal ostemyelitis. Community pneumonia. Staphylococcus aureus. Extrinsic bronquial asthma.

Tordecillas Echenique Y, Salamanca Bautista, MP, Arias Jiménez. JL, Guisado Espartero E, Ortega Calvo M, Pérez. Cano R. Osteomielitis esternal hematógena y neumonía de la comunidad secundarias a una sepsis por Staphylococcus aureus sensible a meticilina. An Med Interna (Madrid) 2005; 22: 191-193.

\section{INTRODUCCIÓN}

La osteomielitis esternal es una enfermedad poco frecuente hoy día $(1,2)$. La mayoría de las veces suele diagnosticarse en drogadictos por vía intravenosa (formas primarias), aunque también puede estar asociada a una aspiración de médula ósea (3) (formas secundarias). Más raro aún es el hecho de que la osteomielitis esternal se presente junto a neumonía grave y sepsis en un contexto de inmunocompetencia. Presentamos a continuación un caso de sepsis estafilocócica ocurrido en un joven con antecedentes de asma bronquial que cursó derrame pleural, lesiones pulmonares y osteomielitis esternal.

\section{CASO APORTADO}

Se trataba de un varón de 27 años de edad, que había sufrido una neumonía siendo niño y que no era fumador, ni tenía hábito etílico.
Estaba diagnosticado de asma bronquial extrínseco y rinoconjuntivitis alérgica con hipersensibilidad a gramíneas y a olivo. Necesitaba de corticoides inhalados de forma esporádica. Tras un esfuerzo físico importante (salir de costalero en una procesión durante unas fiestas locales y haber dormido muy poco) en el verano de 2003 comenzó con dolor en el hemitórax derecho que en ocasiones se le irradiaba al miembro superior derecho y que aumentaba con la inspiración profunda. Posteriormente le apareció fiebre y sensación disneica por lo que acudió a urgencias del hospital. En la exploración clínica del ingreso se le encontró mal estado general y una lesión en fase cicatricial en la pared del abdomen que podía corresponder a un forúnculo. El murmullo vesicular estaba disminuido en la base derecha. Las exploraciones cardiaca y neurológica fueron normales. No había organomegalias.

Presentó leucocitosis (desde $12.000 / \mathrm{mm}$ cúbico hasta 18.700/mm cúbico) con neutrofilia, estando el resto de parámetros hematológicos en límites normales. No había eosinofilia. La creatinina le ascendió durante el ingreso a $1,6 \mathrm{mg} / \mathrm{dl}$ y la urea a $61 \mathrm{mg} / \mathrm{dl}$ Otros valores bioquímicos fueron: bilirrubina total: $2,19 \mathrm{mg} / \mathrm{dl}$, fracción directa: 1,33 mg/dl, LDH: 687 UI/l, GOT: 61 UI/l; GPT: 111 UI/l,

Trabajo aceptado: 2 de diciembre de 2004

Correspondencia: Manuel Ortega Calvo. Avda. de la Cruz del Campo, 36, B1. 1,2²A. 41005 Sevilla. e-mail: ortegacalvo@terra.es 
GGT: 521 UI/l, fosfatasa alcalina 509 UI/l. La velocidad de sedimentación llegó a estar en $71 \mathrm{~mm}$ en la primera hora. La serología para VIH y para los virus B y C de la hepatitis fueron negativas. Se realizaron hemocultivos seriados que resultaron ser positivos para Staphylococcus aureus meticilín sensible.

La ecocardiografía transtorácica mostró imágenes normales sin derrame pericárdico. A los diez días de estar ingresado le apareció una lesión eritematosa y caliente en la región paraesternal derecha que desaparecía a la dígitopresión y que tenía unos 5 x $5 \mathrm{~cm}$. Se aspiró líquido de la lesión cuyo cultivo fue también positivo para $S$. aureus meticilín sensible. La ecografía de abdomen fue normal. En el TAC de tórax había un derrame pleural izquierdo con prominencia intersticial. Se objetivaron tres pequeñas imágenes nodulares en lóbulo superior derecho, lóbulo superior izquierdo y otra de localización subpleural de tamaño menor a un centímetro compatibles con pequeños abscesos estafilocócicos (Fig. 1). El TAC de abdomen fue normal, sin presencia de adenopatías ni líquido en cavidad libre. La gammagrafía ósea mostraba actividad osteogénica intensamente aumentada a nivel de la articulación esternal compatible con artritis a ese nivel. Se le practicó una RMN del esternón. Existía captación de gadolinio en la articulación del manubrio con el cuerpo esternal, extendiéndose la captación hasta la mitad del manubrio y el tercio proximal del cuerpo esternal, con aumento de tamaño y captación asimismo de los tejidos blandos adyacentes, tanto anteriores como posteriores (Fig. 2). Estos hallazgos eran compatibles con artritis a ese nivel y muy sugerentes de osteomielitis. No había signos de destrucción ósea. La evolución clínica del cuadro fue buena con tratamiento antibiótico consistente en cloxacilina intravenosa (IV) durante 15 días a dosis de $2 \mathrm{~g} / 6$ horas y gentamicina IV durante 7 días a dosis de $5 \mathrm{mg} / \mathrm{kg} / 24$ horas. En ningún momento de la evolución necesitó cuidados intensivos. Posteriormente se prosiguió con tratamiento oral a base de rifampicina (dosis de $600 \mathrm{mg} / 24 \mathrm{~h}$ ) y levofloxacino (dosis de $500 \mathrm{mg}$, cada $24 \mathrm{~h}$ ) durante dos meses. La lesión dérmica paraesternal tardó cinco semanas en desaparecer. Para descartar que existiera una inmunodeficiencia primaria se le practicó un despistaje con los resultados siguientes: niveles de complemento, subpoblaciones y marcadores linfocitarios, capacidad oxidativa de granulocitos y funcionalidad linfocitaria normales (esta última con expresión de marcadores de activación en respuesta a distintos estímulos). Niveles protectores para IgG específica frente a toxoide tetánico, Haemophilus $B$ y polisacárido capsular de neumococo. Niveles ligeramente elevados de IgE e IgG4 con normalidad en el resto de inmunoglobulinas y de subclases de $\operatorname{IgG}(\operatorname{IgE}=170 \mathrm{UI} / \mathrm{l}$, rango normal: 0-100 UI/l; Ig G4 $=3.910 \mathrm{mg} / \mathrm{l}$, rango de normalidad: $80-1.400$ $\mathrm{mg} / \mathrm{l}$ ). No se pudieron evaluar los títulos de isohemaglutininas por falta de muestras. Este estudio fue realizado estando el paciente de alta y a los dos meses del comienzo de la enfermedad.

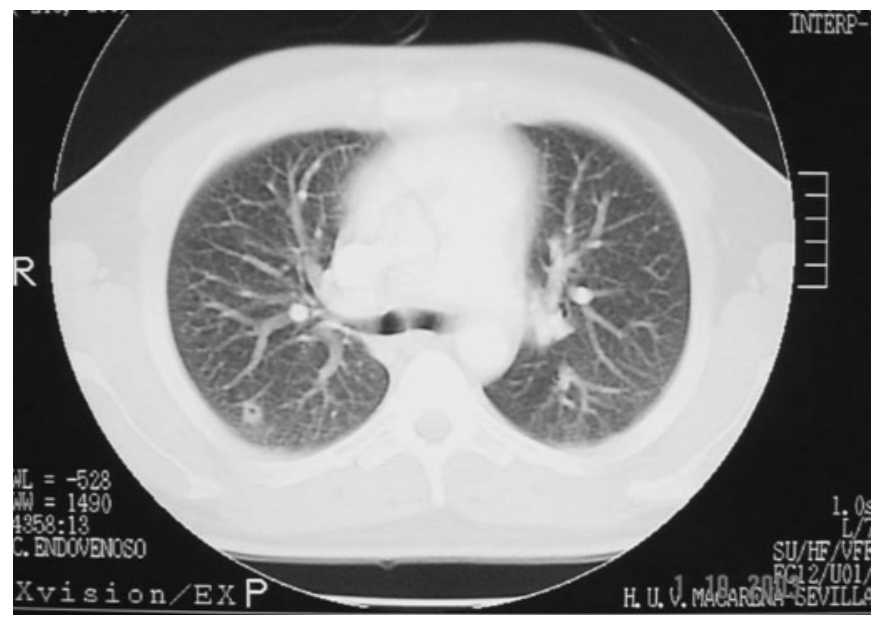

Fig. 1. TAC torácico con una imagen de absceso en el hemitórax derecho compatible con neumonía estafilocócica.

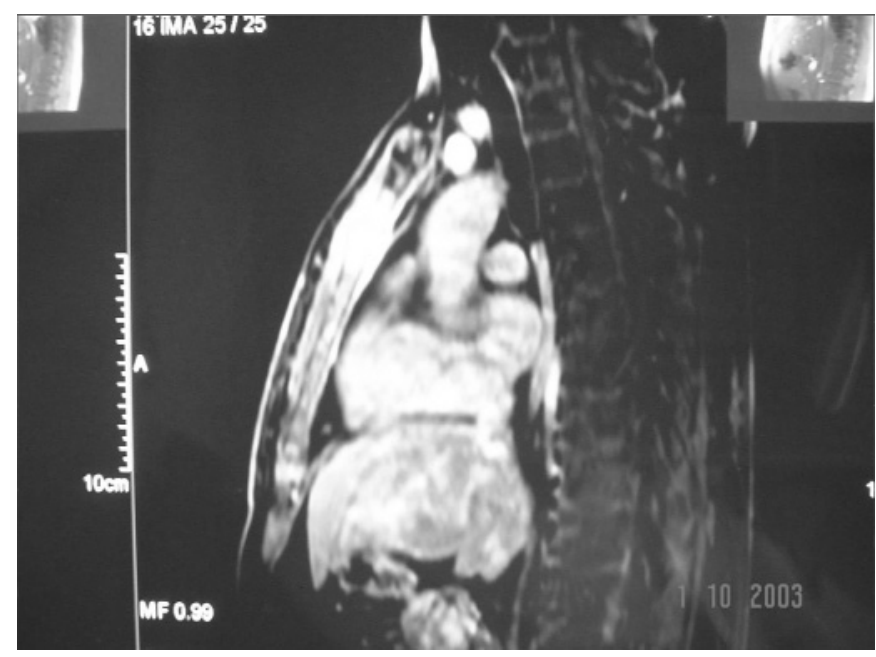

Fig. 2. RMN mostrando una imagen compatible con artritis y osteomielitis en la articulación manubrio-esternal.

\section{DISCUSIÓN}

La mayoría de los casos que se publican de osteomielitis esternal están asociados a traumatismos o a la presencia de un absceso retroesternal $(4,5)$.La patogénesis de la infección invasora por $S$. aureus se debe a la existencia de gran cantidad de proteínas extracelulares (6). Algunas de ellas son secretadas por el propio microorganismo (citoxinas y exoenzimas) mientras que otras permanecen adheridas a la pared celular (proteína A y diversas adhesinas). Estas proteínas le permiten soslayar las defensas del huésped, adherirse a sus células y a las moléculas de la matriz extracelular y expandirse por los tejidos (6). Este mecanismo nos parece el más probable en la génesis de la osteomielitis esternal observada en nuestro paciente. Hay un grupo de enfermedades estafilocócicas no invasoras cuya causa es una toxina única. La más importante de ellas es el síndrome de shock tóxico causado generalmente por la toxina-1 (TSST-1) y menos frecuentemente por otros superantígenos (SAgs) tales como la enterotoxinas A, B, C o D (6).

El estudio con resonancia magnética nuclear es básico para el diagnóstico por imagen de la osteomielitis esternal (7). Aumenta la precocidad del mismo y evita técnicas cruentas como el drenaje quirúrgico. Las imágenes de resonancia que mostraba este paciente eran muy compatibles con el diagnóstico de osteomielitis. La patogenia inmunológica del caso que presentamos puede explicarse por la existencia de un forúnculo en la pared abdominal en fase cicatricial que tras el intenso esfuerzo físico realizado se reactivó y fue capaz de enviar la suficiente cantidad de bacterias al torrente sanguíneo como para crear el cuadro séptico $(8,9)$.

Sutherland y cols. demostraron en un trabajo acerca del efecto de la interleukina-4 sobre la secreción de IgG4 por parte de células mononucleares periféricas (10) que el $S$. aureus de la cepa Cowan I aumentaba su secreción de forma espontánea. Recordemos que este es prácticamente el único rasgo detectado en el estudio inmunológico. También son conocidos los efectos moduladores de TSST-1 sobre la síntesis de IgE en pacientes con dermatitis atópica (11) y la susceptibilidad a infecciones por estafilococo en los pacientes con síndrome 
hiper-IgE (12,13). Arkwright y cols. (14) han demostrado que los niños con dermatitis atópica, portadores de cepas de Estafilococo dorado toxín-positivas, muestran una elevación de los linfocitos B CD5 periféricos sin aumento en la clínica de la enfermedad y sin aumento de los niveles de IgE.

No parece que el hecho de padecer de forma crónica un asma polínico leve-moderado haya sido una causa determinante para el cuadro clínico de este paciente (15). La pauta terapéutica que se le impuso fue coherente con lo publicado para neumonías severas adquiridas en la comunidad (16). Se descartó la existencia de enfermedad granulomatosa crónica mediante el estudio de la función oxidativa de los polimorfonucleares $(17,18)$. La elevación transitoria de urea que presentó el paciente fue debida al cuadro séptico más que a cualquier tipo de complicación renal $(19,20)$.
La rapidez del diagnóstico microbiológico y el aspecto séptico del paciente nos hicieron descartar otras posibilidades casi desde el principio. Por ejemplo, el paciente no tenía aspecto de infección tuberculosa (21), ni de blastomicosis (22) ni de infección por Aspergillus $(17,18,23)$. Se descartó también la osteítis condensante de clavícula (24), el síndrome SAPHO $(25,26)$ y la enfermedad de Lyme $(27)$.

\section{AGRADECIMIENTO}

Los autores agradecen a la Dra. Berta Sánchez Sánchez del Servicio de Inmunología del Hospital Universitario Virgen del Rocío de Sevilla el estudio de despistaje de inmunodeficiencias realizado en este paciente.

\section{Bibliografía}

1. Shukla,PC. Primary sternal osteomyelitis. J Emerg Med 1994; 12: 293297.

2. Mofredj A, Guerin JM, Leibinger F, Masmoudi R. Primary sternal osteomyelitis and septicaemia due to Staphylococcus aureus. Scand J Infect Dis 1999; 31: 98-100.

3. Shah M, Watanakunakorn C. Staphylococcus aureus sternal osteomyelitis complicating bone marrow aspiration. South Med J 1978; 71: 348-349.

4. Cuschieri J, Kralovich KA, Patton JH, Horst HM, Obeid F, Karmy-Jones R. Anterior mediastinal abscess after closed sternal fracture. J Trauma 1999; 47: 551-554.

5. Mallinson RH, Tremlett CH, Payne BV, Richards JE. Sternal osteomyelitis after cardiopulmonary resuscitation. J R Soc Med 1999; 92: 87.

6. Vojtov N, Ross HF, Novick,RP. Global repression of exotoxin synthesis by staphylococcal superantigens. Proc Nat Acad Sci 2002; 99: 10102 10107.

7. Moylett,E,Chung,T,Baker,C.J.: Magnetic resonance imaging in a child with primary sternal osteomyelitis. Pediatr Infect Dis J 2001; 20: 547-550.

8. Friman G, Wesslen L. Special feature for the Olympics: effects of the exercise on the immune system: infections and exercise in high-performance athletes. Immunol Cell Biol 2000; 78: 510-522.

9. Nieman DC. Current perspective on exercise immunology. Curr Sports Med Rep 2003; 2: 239-242.

10. Sutherland M, Blaser K, Pene J. Effects of interleukin-4 and interferongamma on the secretion of IgG4 from human peripheral blood mononuclear cells. Allergy 1993; 48: 504-510.

11. Lester MR, Hofer MF, Renz H, Trumble AE, Gelfand EW, Leung DY. Modulatory effects of staphylococcal superantigen TSST-1 on IgE synthesis in atopic dermatitis. Clin Immunol Immunopathol 1995; 77: 332-338.

12. Ishizaka A, Kojima K, Sakiyama Y, Matsumoto S, Kuwajin K, Wagatsuma Y, et al. Hyper-response of serum IgG1 to Staphylococcus aureus peptidoglycan in patients with hyper-IgE syndrome. Clin Exp Immunol 1992; 1: 53-57.

13. Ishizaka A, Joh K, Shibata R, Wagatsuma MY, Nakanishi M, Tomizawa $\mathrm{K}$, et al. Regulation of IgE and IgG-4 sybthesis in patients with hyper-IgE syndrome. Immunology 1990; 70: 414-416.

14. Arkwright PD, Cookson BD, Haeney MR, Sanyal D, Potter MR, David TJ. Children with atopic dermatitis who carry toxin-positive Staphylococcus aureus strains have an expansion of blood CD5-B lymphocytes wit- hout an increase in disease severity. Clin Exp Immunol 2001; 2: 184-189.

15. To M, To Y, Yamada H, Ogawa C, Otomo M, Suzuki N, et al. Clinical properties of community-acquired pneumonia in patients with asthma. Nihon Kokyuki Gakkai Zasshi 2003; 41: 89-94.

16. De Castro FR, Torres A. Optimizing treatment outcomes in severe community acquired pneumonia. Am J Respir Med 2003; 2: 39-54.

17. Dotis J, Panagopoulou P, Filioti J, Winn R, Toptsis C, Panteliadis C, et al. Femoral osteomyelitis due to Aspergillus nidulans in a patient with chronic granulomatous disease. Infection 2003; 31: 121-124.

18. Barrot E, Ortega-Calvo M, Borderas F, Sánchez J, Melero-Ruiz J, Sánchez-Sánchez B. Bronchocentric granulomatosis as a first clinical manifestation in an adult patient with p67phox deficiency. Respiration 1999; 66: 547-550.

19. Griffin MD, Bjornsson J, Erickson SB. Diffuse proliferative glomerulonephritis and acute renal failure associated with acute staphylococcal osteomyelitis. J Am Soc Nephrol 1997; 8: 1633-1639.

20. Wai AO, Lo AM, Abdo A, Marra F. Vancomycin - induced acute interstitial nephritis. Ann Pharmacother 1998; 32: 1160-1164.

21. Stewart KJ, Ahmed OA, Laing RB, Holmes JD. Mycobacterium tuberculosis presenting as sternal osteomyelitis J R Coll Surg Edinb 2000; 45: 137-137.

22. Saiz P, Gitelis S, Virkus W, Piasecki P, Bengana C, Templeton, A. Blastomycosis of long bones. Clin Orthop 2004; 421: 255-259.

23. Barzaghi N, Emmi V, Mencherini S, Minzioni Marone P, Minoli,L, Sternal osteomyelitis due to Aspergillus fumigatus after cardiac surgery. Chest 1994; 105: 1275-1277.

24. Greenspan A, Gerscovich E, Szabo RM, Matthews JG. Condensing osteitis of the clavicle: a rare but frequently misdiagnosed condition. Am J Roentgenol 1991; 156: 1011-1015.

25. Koh ET. Synovitis, acne, pustulosis, hyperostosis and osteitis (SAPHO) syndrome: a brief review of rare condition. Ann Acad Med Singapore 1998; 27: 122-124.

26. Olivé A, Pérez Andrés R, Rivas A, Holgado S, Casado E, Guma M, et al. El síndrome SAPHO: un estudio de 16 casos. Med Clin (Barc.) 1999; 112: 61-63.

27. De Vega Santos T, Haro Herrero M, Montolio Donate S, Zamarrón Moreno A, Pascual de Pablo E, López López C. Enfermedad de Lyme y síndrome SAPHO. An Med Interna (Madrid) 1997; 14 : 28-30. 